\title{
artigo
}

\section{Estado nutricional e sintomas ansiosos e depressivos em pacientes oncológicos: comparação entre um hospital público e outro particular}

Nutritional state and anxious and depressive symptoms in oncological patients: comparison between a public and private hospital

Estado nutricional y síntomas ansiedos y depresivos en pacientes oncológicos: comparación entre hospital público y privado

\section{RESUMO}

Objetivo: Identificar o perfil nutricional e sintomas ansiosos e depressivos de pacientes oncológicos das redes de atenção à saúde, pública e complementar, e verificar possiveis associações com o estado nutricional. Método: Estudo transversal, realizado no hospital universitário e em um hospital privado de Maceió-AL. Foram mensurados peso, altura, circunferência do braço e circunferência da panturrilha. Para avaliar a presença de sintomas ansiosos e depressivos utilizou-se a Escala Hospitalar de Ansiedade e Depressão. Resultados: A média do IMC foi maior no grupo da rede pública $(p=0,02)$ e a CB no grupo da rede complementar $(p<0,01)$. 0 estado nutricional também foi associado à presença de sintomas ansiosos e depressivos. Os sintomas ansiosos foram associados ao IMC $(p<0,01)$ e a CP $(p<0,01)$. Já os depressivos apenas com o IMC $(p=0,03)$. Conclusão: Observou-se diferenças no estado nutricional dos pacientes nos diferentes serviços, além de apresentar associações com os sintomas de ansiedade e depressão.
\end{abstract}

DESCRITORES: Estado Nutricional; Oncologia; Ansiedade; Depressão.

\begin{abstract}
Objective: Identify the nutritional profile and anxious and depressive symptoms of cancer patients in health care networks, public and complementary, and check for possible associations with nutritional status. Method: Cross-sectional study, carried out at the university hospital and in a private hospital in Maceió-AL. Weight, height, arm circumference, and calf circumference were measured. To assess the presence of anxious and depressive symptoms, the Hospital Anxiety and Depression Scale was used. Results: The mean BMI was higher in the public network group $(p=0.02)$ and the $A C$ in the complementary network group $(p<0.01)$. Nutritional status was also associated with the presence of anxious and depressive symptoms. Anxious symptoms were associated with BMI $(p<0.01)$ and CC $(p<0.01)$. On the other hand, depressives only with BMI $(p=0.03)$. Conclusion: There were differences in the nutritional status of patients in the different services, also being associated with symptoms of anxiety and depression.
\end{abstract}

DESCRIPTORS: Nutritional Status; Medical Oncology; Anxiety; Depression.

\section{RESUMEN}

Objetivo: Identificar el perfil nutricional y los síntomas ansiosos y depresivos de los pacientes oncológicos en las redes de atención pública y complementarias, y verificar posibles asociaciones con el estado nutricional. Método: Estudio transversal, realizado en el hospital universitario y en un hospital privado de Maceió-AL. Se midieron el peso, la altura, la circunferencia del brazo y la circunferencia de la pantorrilla. Para evaluar la presencia de síntomas ansiosos y depresivos se utilizó la Escala Hospitalaria de Ansiedad y Depresión. Resultados: El IMC medio fue mayor en el grupo de la red pública ( $p=0.02$ ) y el CB en el grupo de la red complementaria $(p<0.01)$. El estado nutricional también se asoció con la presencia de síntomas ansiosos y depresivos. Los síntomas de ansiedad se asociaron con el IMC $(p<0,01)$ y la PC $(p<0,01)$. Por otro lado, depresivos solo con IMC $(p=0,03)$. Conclusión: Existieron diferencias en el estado nutricional de los pacientes en los diferentes servicios, además de estar asociadas a síntomas de ansiedad y depresión.

DESCRIPTORES: Estado Nutricional; Oncología Médica; Ansiedad; Depresión.

RECEBIDO EM: 15/12/2020 APROVADO EM: 13/01/2021 


\section{Dafiny Rodrigues Silva}

Nutricionista. Universidade Federal de Alagoas. Maceió/AL - Brasil.

ORCID: 0000-0002-5345-3869

\section{Laís Gomes Lessa Vasconcelos}

Nutricionista. Universidade Federal de Alagoas. Maceió/AL - Brasil.

ORCID: 0000-0002-9445-1966

\section{Natália Mendes de Melo}

Nutricionista. Universidade Federal de Alagoas. Maceió/AL - Brasil.

ORCID: 0000-0001-8784-254X

\section{Janaína da Silva Nascimento}

Nutricionista. Universidade Federal de Alagoas. Maceió/AL - Brasil.

ORCID: 0000-0002-1663-3019

\section{Jayanne Mayara Magalhães de Melo}

Nutricionista. Mestre em Nutrição Humana. Universidade Federal de Alagoas. Maceió/AL - Brasil.

ORCID: 0000-0002-6226-7128

\section{Carlos Queiroz do Nascimento}

Enfermeiro. Doutorando em Saúde e Ambiente. Universidade Tiradentes. Aracaju/SE - Brasil.

ORCID: 0000-0002-3345-2500

\section{João Araújo Barros-Neto}

Nutricionista. Doutor em Processos Interativos dos Órgãos e Sistemas. Universidade Federal de Alagoas. Maceió/AL - Brasil. ORCID: 0000-0002-7603-1095

\section{INTRODUÇÃO}

0 câncer é o principal problema de saúde pública no mundo é classificado como uma das principais causas de morte prematura, antes dos 70 anos, na maioria dos países. ${ }^{1}$ Sua incidência e aumento da taxa de mortalidade, encontram-se em escala crescente, decorrente do envelhecimento populacional e aumento dos fatores predisponentes ao surgimento da doença, especialmente os associados a fatores socioeconômicos e ambientais, tais como: sedentarismo, alimentação inadequada, exposição a poluentes, entre outros ${ }^{2}$

A mais recente estimativa mundial publicada no ano de 2018, aponta que ocorreram 18 milhões novos casos de câncer no mundo e 9,6 milhões de óbitos decorrentes da doença. Sendo o câncer de pulmão acompanhado pelo câncer de mama (2,1 milhóes), cólon e reto (1,8 milhões) e próstata (1,3 milhões), os mais incidentes ${ }^{2}$ Segundo o INCA para o Brasil, a estimativa é que para cada ano do triênio 20202022 ocorrerá 625 mil casos novos de câncer, sendo o câncer de pele não melanoma o mais incidente (177 mil), seguido pelos cânceres de mama e próstata ( 66 mil cada), cólon e reto (41 mil), pulmão $\left(30\right.$ mil) e estômago $(21 \mathrm{mil})^{1}$

Entre os problemas enfrentados pelo paciente oncológico o comprometimento do estado nutricional é um dos mais frequentes, estando presente em $40-80 \%$ dos pacientes ao longo da doença. Este agravo atinge em torno de $15-20 \%$ dos indivíduos no momento do diagnóstico e pode chegar até $90 \%$ dos pacientes com estágio mais avançado da doença ${ }^{3}$

O comprometimento do estado nutricional ocorre devido às alterações metabólicas causadas pelo tumor, que compete por nutrientes para manter-se em crescimento e para isto leva a anormalidades no metabolismo dos macronutrientes (carboidratos, proteínas e lipídeos) ${ }^{4}$. Podemos também associar o tipo de tratamento à qual o paciente está sendo submetido, quimioterápico e/ou radioterápico, que apresentam efeitos colaterais como náuseas, vômitos, estomatite, mucosite, diarreia e constipação, que contribuem para a diminuição da ingestão alimentar, resultando em déficit energético e balanço nitrogenado negativo ${ }^{3,4}$

Nestas condições, onde o declínio do estado nutricional é um dos efeitos colaterais mais impactantes ao paciente, sintomas psicológicos podem ser desencadeados devido a perspectivas que o indivíduo pode desenvolver sobre suas chances de cura. Sendo a ansiedade e depressão a mais frequentes neste público, podendo acometer cerca de um terço dos pacientes em tratamento ${ }^{5}$

Repercussões adversas como menor adesão ao tratamento, maior tempo de hospitalização, piora da qualidade de vida, piora do prognóstico e sobrevida, estão mais presentes nos indivíduos que desenvolvem sintomas de ansiedade e depressão ${ }^{5,6}$ Um estudo realizado pela National Comorbidity Survey mostrou que 51\% dos pacientes diagnosticados com depressão e ansiedade manifestaram exacerbação de sintomas, tempo de recuperação prolongada e mais readmissões em serviço de saúde ${ }^{6}$

Diante do exposto o presente estudo teve como objetivo identificar o perfil nutricional e sintomas ansiosos e depressivos de pacientes em tratamento quimioterápico em hospitais de diferentes redes de atenção à saúde (pública e complementar) de Maceió - AL, bem como identificar e possíveis associações entre característica do acesso ao serviço de saúde e a presença 


\section{artigo}

Rodrigues, D.R.; Vasconcelos, L.G.L.; Melo, N.M.; Nascimento, J.S.; Melo, J.M.M.; Nascimento, C.Q.; Barros-Neto

Estado nutricional e sintomas ansiosos e depressivos em pacientes oncológicos: comparação entre um hospital público e outro particular

de sintomas ansiosos e depressivos com o estado nutricional desses pacientes.

\section{MÉTODOS}

A presente pesquisa foi submetida e aprovada na Plataforma Brasil e ao Comitê de Ética em Pesquisa da Universidade Federal de Alagoas - UFAL sob o número CAAE 52019315.0.0000.5641. Trata-se de um estudo transversal, realizado com pacientes em tratamento adjuvante (quimioterapia) advindos de um hospital universitário da rede pública de saúde e de um hospital da rede complementar de saúde de Maceió, no período de novembro de 2015 a janeiro de 2016. A amostra foi composta por um plano de amostragem não probabilística de conveniência, com presença de indivíduos de ambos os sexos, com idade superior a 18 anos, diagnosticados com câncer em qualquer região, que estavam em tratamento quimioterápico exclusivo, e que aceitaram assinar o termo de consentimento livre esclarecido (TCLE).

A coleta de dados foi realizada por graduandos em nutrição, devidamente treinados. Foram aplicados formulários estruturados contendo variáveis sociodemográficas e antropométricas. $\mathrm{O}$ estado nutricional foi avaliado utilizando-se peso, altura, circunferência do braço (CB) e circunferência da panturrilha (CP).

Para a coleta do peso foi utilizada balança do tipo plataforma digital, calibrada, com capacidade para $200 \mathrm{Kg}$ e resolução de $100 \mathrm{~g}$. Os indivíduos foram pesados sem sapatos e sem adornos, mantendo-se em posição ortostática.

Para os indivíduos com 60 anos ou mais, foi estimatimada a estatura a partir da mensuração da altura do joelho, com o auxílio de paquímetro, e aplicada em fórmula, segundo Chumlea et al.7. Para os demais indivíduos, foi realizada a aferição da estatura utilizando-se estadiômetro do tipo vertical, portátil, de piso, com faixa de medição de 0 a $200 \mathrm{~cm}$ e graduação milimétrica, com o indivíduo em pé, descalço, encostando região occipital, clavícula, nádegas, panturrilha e calcanhares no aparelho, mantendo a cabeça (sem adornos) ereta e com o olhar fixo à frente (plano horizontal de Frankfurt); baixava-se o cursor e a leitura do valor obtido era feita.
O índice de massa corporal (IMC) foi classificado a partir da divisão do peso (em quilogramas) pela estatura ao quadrado (em metros), adotando-se o critério de Lipschitz ${ }^{8}$, para os indivíduos com 60 anos ou mais e a classificação adotada pela Organização Mundial de Saúde9 para indivíduos com idade inferior a 60 anos.

Para mensuração da CP e CB foi utilizada fita métrica inelástica para medidas antropométricas (escala de $0,1 \mathrm{~mm}$ ). A medida da CP foi realizada com o indivíduo sentado, posicionando a perna em ângulo de $90^{\circ}$ e pés apoiados no chão, sendo então considerado o ponto de maior circunferência.

Já a medida da CB foi avaliada com o pesquisador posicionando-se atrás do avaliado e solicitando ao indivíduo flexionar seu cotovelo em $90^{\circ}$, com a palma da mão voltada para cima. Por meio de palpação, eram localizados os pontos mais distais do acrômio e olécrano, passava-se a fita e então era feita uma pequena marcação do ponto médio entre estas duas extremidades. Ainda em posição ereta era solicitado ao indivíduo deixar o braço relaxado e estendido ao longo do corpo. No ponto previamente marco, era feita a medida da circunferência do braço com posterior registro do valor obtido.

Para avaliação da presença de sintomas ansiosos e depressivos foi utilizada a Escala Hospitalar de Ansiedade e Depressão (HADS), que visa detectar graus leves de transtornos afetivos em ambientes não psiquiátricos e é constituída por 14 itens de múltipla escolha, dos quais sete são voltados para avaliação da ansiedade (HADS-A) e sete para depressão (HADS-D) ${ }^{10}$

Os dados coletados foram organizados em banco de dados eletrônicos no Programa Estatístico SPSS (Statistical Package for Social Sciences) em sua versão $20.0^{\circ}$, os quais foram apresentados por meio de estatística descritiva de frequência e medidas de tendência central (média + desvio padrão). Foi realizada avaliação da distribuição paramétrica das amostras por meio do teste Shapiro-Wilk. Todas as variáveis analisadas apresentaram distribuição paramétrica. $\mathrm{O}$ teste-t de student para amostras independentes foi utilizado para verificar diferença entre as médias de duas variáveis, adotando-se um $\alpha=5 \%$.

\section{RESULTADOS}

A amostra foi composta por 65 indivíduos assistidos por dois hospitais de Maceió, sendo $50,8 \%(\mathrm{n}=33)$ provenientes de um hospital público e 49,2\% ( $n=32)$ de um hospital particular, com média de idade 54,2 anos, sendo um terço desses indivíduos idosos. Comparando os indivíduos do hospital da rede pública vs. hospital particular, observou as respectivas médias de IMC 27,49 $\pm 4,62 \mathrm{~kg} /$ $\mathrm{m}^{2}$ vs. $24,79 \pm 4,05 \mathrm{~kg} / \mathrm{m}^{2}(\mathrm{p}=0,02)$; circunferência da panturrilha $34,13 \pm 4,20 \mathrm{~cm}$ vs. $33,08 \pm 3,05 \mathrm{~cm}(\mathrm{p}=0,687)$; e circunferência do braço média de $27,53 \pm 3,40 \mathrm{~cm}$ vs. 30,65 $\pm 2,77 \mathrm{~cm}(\mathrm{p}<0,01)$; respectivamente (tabela 1). A média de renda entre os grupos foi de $\mathrm{R} \$ 1380,00$ vs. $\mathrm{R} \$ 2011,66$ ( $\mathrm{p}=0,026$ ).

A presença de sintomas de ansiedade resultando em provável diagnóstico foi observada em 50,8\% ( $n=33)$ da amostra e em relação à depressão, 52,3\% (n=34) apresentavam possível diagnóstico (Tabela 1).

A média do IMC foi maior no grupo da rede pública, classificando este grupo com excesso de peso. Enquanto que um dos indicadores de melhor reserva muscular, como a $\mathrm{CB}$, apresentou melhores resultados no grupo de pacientes da rede complementar de saúde, evidenciando que quanto melhor a renda e a escolaridade, em pacientes com câncer, melhor o autocuidado com o estado nutricional e composição corporal.

A presença de sintomas ansiosos e depressivos foi muito mais frequente entre os pacientes que realizavam tratamento no hospital particular (rede complementar de saúde), motivo pelo qual mereceu destaque para as análises seguintes de avaliação do estado nutricional.

Assim, comparando o estado nutricional entre os grupos de indivíduos com provável vs. improvável diagnóstico de ansiedade, observou-se que o provável diagnóstico de ansiedade associou-se com o IMC ( $\mathrm{p}$ $<0,01$ ), com média de $22,08 \mathrm{~kg} / \mathrm{m}^{2}$, enquanto que os indivíduos com diagnóstico improvável apresentaram IMC médio de $25,26 \mathrm{~kg} / \mathrm{m}^{2}$. A CP também apresentou associação $(p<0,01)$, onde o grupo com provável diagnóstico apresentou circunferência média de $32,20 \mathrm{~cm}$ vs $35,02 \mathrm{~cm}$, do grupo com diagnóstico improvável. Não 
Tabela 1. Características socioeconômicas e de saúde dos indivíduos em tratamento adjuvante (quimioterapia), segundo característica do serviço de saúde. Maceió, 2016 ( $n=65)$.

\begin{tabular}{|c|c|c|c|c|c|}
\hline & \multicolumn{2}{|c|}{ PÚBLICO (N = 33) } & \multicolumn{2}{|c|}{ PRIVADO ( $N=32$ ) } & \multirow{2}{*}{$\mathbf{P}$} \\
\hline & $\mathrm{N}$ & $\%$ & $\mathrm{~N}$ & $\%$ & \\
\hline \multicolumn{6}{|l|}{ Sexo } \\
\hline Feminino & 22 & 66,67 & 22 & 68,75 & \multirow{2}{*}{$0,86^{a}$} \\
\hline Masculino & 11 & 33,33 & 10 & 31,25 & \\
\hline \multicolumn{6}{|l|}{ Idade (anos) } \\
\hline$<60$ anos & 20 & 60,61 & 25 & 78,125 & \multirow{2}{*}{$0,13^{\mathrm{a}}$} \\
\hline$\geq 60$ anos & 13 & 39,39 & 7 & 21,875 & \\
\hline \multicolumn{6}{|l|}{ Estado Civil } \\
\hline Solteiro/ divorciado/ viúvo & 18 & 54,55 & 13 & 40,625 & \multirow{2}{*}{$0,26^{a}$} \\
\hline União estável / casado & 15 & 45,45 & 19 & 59,375 & \\
\hline \multicolumn{6}{|l|}{ Anos de estudo } \\
\hline$\leq 5$ anos & 19 & 57,58 & 5 & 15,625 & \multirow{2}{*}{$<0,01^{\mathrm{a}}$} \\
\hline$>5$ anos & 14 & 42,42 & 27 & 84,375 & \\
\hline \multicolumn{6}{|l|}{ Ansiedade } \\
\hline Improvável & 26 & 78,79 & 6 & 18,75 & \multirow{2}{*}{$<0,01^{\mathrm{a}}$} \\
\hline Provável & 7 & 21,21 & 26 & 81,25 & \\
\hline \multicolumn{6}{|l|}{ Depressão } \\
\hline Improvável & 26 & 78,79 & 5 & 15,625 & \multirow{2}{*}{$<0,01^{2}$} \\
\hline \multirow[t]{2}{*}{ Provável } & 7 & 21,21 & 27 & 84,375 & \\
\hline & Média & $\mathrm{DP}$ & Média & DP & $p$ \\
\hline IMC & 27,49 & 4,62 & 24,79 & 4,05 & $0,02^{\mathrm{b}}$ \\
\hline $\mathrm{CP}$ & 34,13 & 4,2 & 33,08 & 3,05 & $0,29^{b}$ \\
\hline CB & 27,53 & 3,4 & 30,65 & 2,77 & $<0,01^{b}$ \\
\hline
\end{tabular}

Tabela 2. Classificação do estado nutricional dos indivíduos em tratamento adjuvante (quimioterapia), segundo característica do serviço de saúde. Maceió, 2016 (n=65).

\begin{tabular}{|c|c|c|c|c|c|}
\hline & \multicolumn{2}{|c|}{$\begin{array}{l}\text { IMPROVÁVEL } \\
\text { ANSIEDADE }\end{array}$} & \multicolumn{2}{|c|}{$\begin{array}{l}\text { PROVÁVEL } \\
\text { ANSIEDADE }\end{array}$} & \multirow[t]{2}{*}{$\mathbf{P}$} \\
\hline & MÉDIA & $\mathrm{DP}$ & MÉDIA & $\mathrm{DP}$ & \\
\hline IMC & 28,02 & 4,57 & 24,26 & 3,66 & $<0,01^{a}$ \\
\hline $\mathrm{CP}$ & 35,02 & 3,99 & 32,20 & 2,86 & $<0,01^{a}$ \\
\hline \multirow[t]{3}{*}{ CB } & 28,78 & 3,9 & 29,09 & 3,04 & $0,74^{a}$ \\
\hline & \multicolumn{2}{|c|}{ Improvável depressão } & \multicolumn{2}{|c|}{ Provável depressão } & D \\
\hline & Média & DP & Média & DP & 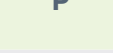 \\
\hline IMC & 27,37 & 4,3 & 24,98 & 4,48 & $0,03^{a}$ \\
\hline $\mathrm{CP}$ & 34,54 & 4,07 & 32,71 & 3,16 & $0,63^{a}$ \\
\hline CB & 28,67 & 3,84 & 29,21 & 3,09 & $0,55^{a}$ \\
\hline
\end{tabular}

apresentando associação em relação ao $\mathrm{CB}$ $(\mathrm{p}=0,74)$ (tabela 2).

No que se refere a presença de sintomas depressivos, o grupo com provável diagnóstico apresentou IMC inferior $(\mathrm{IMC}=22,37$ $\left.\mathrm{kg} / \mathrm{m}^{2}\right)$ ao grupo com diagnóstico improvável $\left(\mathrm{IMC}=24,98 \mathrm{~kg} / \mathrm{m}^{2}\right)(\mathrm{p}=0,03)$. Não sendo possível observar diferença entre as médias da $\mathrm{CP}$ e da $\mathrm{CB}$, entre os indivíduos com ou sem provável diagnóstico (tabela 2).

\section{DISCUSSÃO}

Resultados similares foram encontrados por Chabowski et al. ${ }^{11}$ em um estudo com 257 pacientes portadores de câncer de pulmão, onde descobriu-se que dois terços dos pacientes, aproximadamente $65 \%$, apresentavam diferentes graus de transtornos depressivos e ansiosos de acordo com a escala HADS. Ainda, relações semelhantes foram encontradas entre o estado nutricional e o escore HADS, em que o melhor estado nutricional foi associado a níveis mais baixos de ansiedade e depressão $(\mathrm{r}=-0,68 ; \mathrm{p}$ $<0,001$ er $=-0,60 ; p<0,001$, respectivamente).

Vale ressaltar que são várias as características próprias do câncer e do seu tratamento que podem afetar o equilíbrio mental e fisico do indivíduo ${ }^{12}$ Pitman et al. ${ }^{13}$ referem que existem duas principais vias pelas quais a depressão e a ansiedade podem surgir em pacientes com câncer: os processos envolvidos no modelo biopsicossocial e a diversidade de efeitos neuropsiquiátricos específicos de certos tipos de câncer e seus tratamentos.

Quanto ao meio biopsicossocial, a depressão e a ansiedade surgem mais comumente de reações psicológicas do paciente ao diagnóstico, às perdas inerentes ao tratamento (como queda cabelo, diminuição da função sexual ou de outros órgãos), expectativas em relação a sua sobrevivência e efeitos sobre o trabalho e os papéis sociais, levando a períodos prolongados de estresse ${ }^{14}$

Diante de períodos de estresse sustentados, a sintomatologia da depressão e ansiedade pode surgir como consequência de efeitos fisiológicos, como ativação do eixo hipotálamo-hipófise-adrenal. Outras situações estressantes como o impacto físico e efeitos colaterais das quimioterapias convencionais (como vômitos, queda de cabe- 


\section{artigo}

Rodrigues, D.R.; Vasconcelos, L.G.L.; Melo, N.M.; Nascimento, J.S.; Melo, J.M.M.; Nascimento, C.Q.; Barros-Neto

Estado nutricional e sintomas ansiosos e depressivos em pacientes oncológicos: comparação entre um hospital público e outro particular

lo, mucosite e neuropatia periférica) podem intensificar os sintomas psicológicos ${ }^{13}$.

O mau estado psicológico de pacientes com câncer pode agravar seu estado nutricional, por meio da diminuição de sua ingestão energética, impactando no prognóstico e mortalidade da doença, devido a associação com a baixa qualidade de vida, menor nível de atividade, aumento dos sintomas adversos relacionados ao tratamento e redução da resposta do tumor à terapia ${ }^{15}$.

Junto a isso, soma-se a perda de peso que pode ocorrer como resultado da elevada demanda energética causada pela inflamação e catabolismo da própria doença ou absorção prejudicada de nutrientes devido a obstruçôes gastrointestinais por tumores, disfagia, dor, anorexia, saciedade precoce, diarréia, hemorróidas, fissuras anais e modificações no cheiro e no paladar, entre outros ${ }^{16}$.

Todas essas repercussões que podem estar presentes em pacientes acometidos pelo câncer, associadas às alterações metabólicas e estilo de vida dos mesmos, podem contribuir para perda de peso e consequentemente a diminuição do IMC, assim como da circunferência da panturrilha, sendo esta última considerada como um indicador sensível de alterações musculares no indivíduo, especialmente em idosos, e deve ser utilizada para monitoração dessas alterações ${ }^{17}$.

Observa-se então que a nutrição deve atuar no sentido de promoção da saúde, prevenção de demais agravos e auxiliadora no tratamento, buscando garantir formas de atingir as necessidades nutricionais do paciente, para preservar o peso e a composição corporal, bem como desacelerar o surgimento e progressão da caquexia, contribuindo com orientações para o controle de sintomas, possibilitando a redução da ansiedade e aumento do prazer e da autoestima ${ }^{18}$.

\section{CONCLUSÃO}

A partir do presente estudo pode-se identificar elevada frequência de pacientes com câncer com possível/provável diagnóstico de ansiedade e depressão. A presença de sintomas ansiosos foi associada ao comprometimento do estado nutricional (avaliado pelo IMC e CP).

Dessa forma, enfatiza-se a importância da avaliação nutricional e psicológica para identificação precoce de fatores que contribuem com a piora da progressão da doença de pacientes com câncer, visando aprimorar o manejo do tratamento e melhoria da qualidade de vida do mesmo, bem como uma melhor resposta ao tratamento proposto.

\section{REFERÊNCIAS}

1. INSTITUTO NACIONAL DE CÂNCER JOSÉ ALENCAR GOMES DA SILVA. Estimativa 2020: incidência de câncer no Brasil. [publicação online]; 2019. [acesso em DIA mês 2020]. Disponível em: https:// www.inca.gov.br/sites/ufu.sti.inca.local/files//media/document// estimativa-2020-incidencia-de-cancer-no-brasil.pdf

2. Bray F, Ferlay J, Soerjomataram I, Siegel RL, Torre LA, Jemal A. Global cancer statistics 2018: GLOBOCAN estimates of incidence and mortality worldwide for 36 cancers in 185 countries. CA: a cancer journal for clinicians. 2018;68(6):394-424.

3. Pastore CA, Oehlschlaeger MHK, Gonzalez MC. Impacto do estado nutricional e da força muscular sobre o estado de saúde geral e qualidade de vida em pacientes com câncer de trato gastrintestinal e de pulmão. Revista Brasileira de Cancerologia. 2013;59(1):43-49.

4. Poltronieri T S, Tusset C. Impacto do tratamento do câncer sobre o estado nutricional de pacientes oncológicos: atualização da literatura. Rev. bras. ciênc. Saúde. 2016;20(4):327-332.

5. Avelar AMA, Derchain SFM, Camargo CPP, Sarian LOZ, Yoshida A. Qualidade de vida, ansiedade e depressão em mulheres com câncer de mama antes e após a cirurgia. Revista de ciências médicas. 2012;15(1):11-20.

6. Bergerot CD, Laros JA, De Araujo TCCF. Avaliação de ansiedade e depressão em pacientes oncológicos: comparação psicométrica. Psico-USF. 2014;19(2):187-197.

7. Chumle WC, Roche AF, Steinbaugh ML. Estimating stature from knee height for persons 60 to 90 years of age. Journal of the American Geriatrics Society. 1985;33(2):116-120.

8. Lipschitz DA. Screening for nutritional status in the elderly. Primary Care. 1994;21(1):55-67.

9. WORLD HEALTH ORGANIZATION. Obesity: preventing and managing the global epidemic. Report of a World Health Organization
Consultation. Geneva: World Health Organization. [publicação online]; 2000. p. 253. [acesso em dia mês 2020]. Disponível em: https:// www.who.int/nutrition/publications/obesity/WHO_TRS_894/en/

10. Zigmond AS, Snaith RP. The hospital anxiety and depression scale. Acta psychiatrica scandinavica. 1983;67(6):361-370.

11. Chabowski M, Polański J, Jankowska-Polańska B, Janczak D, Rosińczuk J. Is nutritional status associated with the level of anxiety, depression and pain in patients with lung cancer?. Journal of thoracic disease. 2018;10(4):2303-2310.

12. Costa Al, Chaves MD. Perception of anxiety in cancer patients under chemotherapy. Rev. enferm. UFPE on line. 2014;8(3):649653.

13. Pitman A, Suleman S, Hyde N, Hodgkiss A. Depression and anxiety in patients with cancer. Bmj. 2018;361:k1415.

14. Smith HR. Depression in cancer patients: Pathogenesis, implications and treatment. Oncology letters. 2015;9(4):1509-1514.

15. Mantzorou M, Koutelidakis A, Theocharis S, Giaginis C. Clinical value of nutritional status in cancer: what is its impact and how it affects disease progression and prognosis?. Nutrition and Cancer. 2017;69(8):1151-1176.

16. Citak E, Zeliha T, Uzel O. Nutritional status in patients with head and neck cancer undergoing radiotherapy: a longitudinal study. Support Care Cancer. 2019;27(1):239-247.

17. Borges LR, Paiva SI, Silveira DH, Assunção MCF, Gonzalez MC. Can nutritional status influence the quality of life of cancer patients?. Revista de Nutrição. 2010;23(5):745-753.

18. Morais SRD, Bezerra AN, Carvalho NSD, Viana ACC. Nutrição, qualidade de vida e cuidados paliativos: uma revisão integrativa. Revista Dor. 2016;17(2):136-140. 